Research Article

\title{
Optimizing Disruption Recovery Operations for Wind Farms considering Power Generation Loss and Repair Time Uncertainty
}

\author{
Dongmei Zhang $\mathbb{D}^{1},{ }^{1}$ Jiangang Jin $\mathbb{D}{ }^{2}$ Qingchang Ji $\mathbb{D}^{1},{ }^{1}$ and Xintong Zhang ${ }^{3}$ \\ ${ }^{1}$ School of Automation and Communication Engineering, Hebei University of Water Resources and Electric Engineering, \\ Hebei, China \\ ${ }^{2}$ School of Naval Architecture, Ocean \& Civil Engineering, Shanghai Jiao Tong University, Shanghai, China \\ ${ }^{3}$ PowerChina Hebei Electric Power Design \& Research Institute Co., Ltd., Hebei, China \\ Correspondence should be addressed to Jiangang Jin; jinjg05@gmail.com
}

Received 3 August 2019; Revised 4 February 2020; Accepted 7 February 2020; Published 10 March 2020

Academic Editor: Benjamin Ivorra

Copyright (c) 2020 Dongmei Zhang et al. This is an open access article distributed under the Creative Commons Attribution License, which permits unrestricted use, distribution, and reproduction in any medium, provided the original work is properly cited.

\begin{abstract}
The development of wind power in China shows a dramatic growth in the past decade in terms of installed capacity. However, wind power companies mainly focus on the construction of new wind farms continuously, while operations management once wind farms are built is seldom paid attention to. The problem is crucial for ensuring efficient power generation, especially when wind turbines' performance declines over time and disruption/failure often occurs. Efficient disruption recovery operations are critical for restoring the failures of wind turbine generators as fast as possible. This paper aims to optimize the disruption recovery operations for wind farms by determining the maintenance schedule and route for multiple maintenance teams. This optimization problem is formulated as a deterministic mixed integer linear programming model with the objective of minimizing the loss of power generation due to failure. In view of the high uncertainty of repair time, a chance-constrained programming model and a cutting-plane solution algorithm are further proposed. A case study based on a real wind farm demonstrates (1) the proposed model is applicable for solving real-world-sized problems; (2) the optimal maintenance route often shows a crossing pattern, which is quite different from that of traditional vehicle routing problems; and (3) the working time limit violation for maintenance teams due to uncertain repair time can be effectively avoided. Overall, the proposed optimization model provides decision-making support for wind farm maintenance work and shows a great potential in wind farm energy management.
\end{abstract}

\section{Introduction}

To cope with the challenge of global climate change, energy transformation has emerged to be the most critical and urgent task for countries all over the world. With the Paris Climate Agreement, green and renewable energy development has been highly concerned by the international society. As the representative of renewable energy source, wind power has been developed as a renewable energy power generation technology and grown very quickly in the world $[1,2]$. Wind power generation has turned to be a large-scaled and well-commercialized industry and contributed greatly to the sustainable energy development. Up to now, more than 90 countries have built wind power generation systems, among which 29 counties have reached $1 \mathrm{GW}$ installed capacity and 9 countries have exceeded $10 \mathrm{GW}$. The Renewables 2018 Global Status Report (GSR) which was released by "Renewable Energy Policy Network for the 21st Century (REN21)" stated that, in 2017, the newly installed capacity of wind power in the world reached $52 \mathrm{GW}$ and the accumulative installed capacity was $539 \mathrm{GW}$. In the past one decade, China has experienced a rapid growth in wind power generation system development and taken the leading role in global wind power development. China's wind energy capacity will reach at least $150 \mathrm{GW}$ or possibly up to $230 \mathrm{GW}$, which could cut the carbon dioxides by 410 million tons of 
$\mathrm{CO}_{2}$ emission or 150 million tons of coal consumption in energy systems in different scenarios by 2020 . This positive projection of $230 \mathrm{GW}$ of wind energy is equal to 13 times the capacity of the Three Gorges Dam, with the ability to replace power generated from 200 coal fire power plants [3].

However, the rapid development of wind power generation in China mainly focuses on launching new wind power generation projects and installing new wind turbine generators (WTGs), while operations management for existing wind power generation systems once built is seldom paid attention to. This is due to the following reasons: (1) the larger installed capacity of wind farms is accomplished, and the more income and feed-in-tariff could be obtained; (2) most of the wind power generation projects are relatively new, and the WTGs are within the maintenance service period by the manufacturer; and (3) disruption/failure is scarce in the early stage, and maintenance has not become an urgent task for the operators. However, the operation and maintenance problem is crucial for ensuring efficient power generation, especially when wind turbines' performance declines over time (usually after 3-5 years) and disruption/ failure often occurs. Efficient disruption recovery operations are critical for restoring the failures of wind turbine generators as fast as possible. Therefore, it is imperative to optimize the disruption recovery operations for wind farms with the objective of minimizing power generation loss. Specifically, given a number of WTGs in a wind farm that need to be repaired, we need to determine the optimal maintenance schedule and route for maintenance teams.

The operations and management of wind power generation systems is expected to be more and more important and challenging in the near future. With a longer service time, disruption/failure of the WTGs would become more frequent and costly. The natural environment where the wind power is located at is very severe, and it may encounter strong wind, sandstorm, salt fog, wind storm, snow storm, and thunder storm. All these meteorological disasters compose a challenge for WTGs, and sometimes, they cause damage to WTGs. Particularly, some hidden dangers may appear gradually after the WTGs are operated for three to five years. Effectiveness management strategy and efficient disruption recovery operations could help reduce power generation loss once significant disruption happens in a wind farm. Therefore, it is necessary to study the recovery operation planning problem for handling the disruption/ failure of WTGs and optimize the maintenance task decisions, including the maintenance routing and scheduling, so that the power generation loss due to disruptions could be minimized.

In this paper, we aim to address the disruption/failure recovery operation planning problem with the objective of minimizing power generation loss. The contribution lies in the proposed optimization models which can be applied for the decision-making process of wind farm maintenance work and show a great potential in wind farm energy management. Specifically, we contribute to the following:

(1) Proposing a deterministic mixed integer linear programming model for the studied problem
(2) Developing a chance-constrained programming model as well as a cutting-plane-based solution algorithm with consideration of the repair time uncertainty

(3) Demonstrating through case studies that the proposed optimization model could be solved efficiently and is applicable for real-world-sized problems

(4) Finding unique features of the recovery operation routing pattern which is helpful for the wind farm maintenance operators and service providers

We remark that the methodology employed in this study is mainly based on mixed integer linear programming and chance-constrained technique, rather than metaheuristic approaches (e.g., genetic algorithm). This is mainly because linear programming models yield optimal solutions, while metaheuristic approaches only find near-optimal ones and cannot guarantee optimality.

The rest of this paper is organized as follows: Section 2 briefly reviews relevant literature. A detailed problem description is presented in Section 3, and then Section 4 develops mathematical optimization models. A case study is discussed in Section 5. Finally, Section 6 presents the concluding remarks and potential research directions.

\section{Literature Review}

The operations of wind power generation systems involve various decision-making problems and have attracted much attention from researchers. Earlier studies developed optimization models and algorithms to assist decision-making in wind turbine location [4], cable routing [5], power generation forecasting [6,7], failure analysis [8], disruption and maintenance operations $[9,10]$, etc. Methodologies mainly include mixed integer linear programming, game theory, fuzzy theory, and heuristic algorithms $[5,11,12]$.

Disruption and maintenance operations for wind power generation systems have been gaining increasing attention recently. Focusing on preventive maintenance, Perez-Canto and Rubio-Romero integrated the wind farm with traditional electric generating systems and developed an optimization model for scheduling the power plant preventive maintenance works [13]. The postdisruption maintenance problem is quite critical, and various methods have been proposed for finding the optimal routes and schedules for maintenance vehicles, vessels, and personnel [14-17]. Stalhane et al. proposed two formulations, an arc-flow and a path-flow formulation, for scheduling the maintenance tasks at offshore wind farms [14]. Dai et al. considered the temporal dimension and proposed mathematical formulations for the routing and scheduling problem offshore wind farms, to determine the maintenance works of each day over the planning period [15]. Irawan et al. studied a similar problem and developed an algorithm based on Dantzig-Wolfe decomposition methods. An integer linear programming model was proposed to find the optimal route configuration along with the maintenance schedules with the objective of minimizing travel and technician costs [16]. $\mathrm{Bao}$ and $\mathrm{Li}$ investigated a general maintenance operation 
scheduling problem with consideration of random machine breakdowns. They proposed a combined scheduling strategy which adjusts the scheme to improve the robustness of the decision scheme [17]. Disruption recovery operation planning in other contexts has been also studied, such as airline [18], public transportation [19], and humanitarian logistics [20]. We remark that earlier studies mainly treat maintenance working time as fixed and known values without consideration of repair time uncertainty. However, we observe from the practice that the repair time for WTGs is highly uncertain, leading to frequent excessive working time than required for maintenance teams. The WTG repair time can only be known once the maintenance work is finished. Therefore, it is necessary to take maintenance time uncertainty into consideration when planning the maintenance operations.

The studied disruption recovery operation planning problem resembles vehicle routing problems. However, vehicle routing problems are often studied in other contexts, such as logistics delivery [21-23], maritime shipping [24-26], and healthcare management [27-29]. Abundant optimization models and solution algorithms have been developed accordingly. Grangier et al. focused on twoechelon multitrip vehicle routing problem with satellite synchronization and developed an adaptive large neighbourhood search algorithm. Efficient heuristic techniques for destruction, repair, and move operations were proposed [21]. Cirovic et al. studied the logistics vehicle routing problem in urban areas and proposed a model combining simulated annealing, neuro-fuzzy logic, and modified Clarke-Wright algorithm [22]. Ehmke and Mattfeld studied the vehicle routing for attended home deliveries and presented a heuristic optimization framework with consideration of time-dependent travel time data. Zhou et al. introduced a new city logistics problem arising in the last mile distribution, as is called multidepot two-echelon vehicle routing problem with delivery operations [23]. A hybrid multipopulation genetic algorithm was developed to solve the problem. Christiansen et al. conducted an in-depth overview of existing models and solution methods for the linear shipping network design, including mixed integer programming models and two-stage algorithms [24]. Kjeldsen summarized ship routing and scheduling problems in the liner shipping industry and discussed unique features of maritime shipping [25]. Wibisono and Jittamai proposed a biobjective evolutionary algorithm for a liner shipping network design problem and designed a genetic algorithmbased solution method [26]. Fikar and Hirch presented a comprehensive overview on home-healthcare routing and scheduling problems, in which nurses and doctors are scheduled and routed to visit multiple patients' homes [27]. Mankowska et al. studied the home-healthcare routing and scheduling problem with particular consideration of the interdependencies between two services [28]. A mathematical model formulation was proposed, and a powerful heuristic based on a sophisticated solution representation was designed. Begur et al. integrated stand-alone PC-based geographic-information-system software with scheduling heuristics and databases and developed a decision-support system for scheduling home-healthcare nurses [29]. Significant travel time and schedule-preparation time can be saved, and balance of work among nurses can also be improved. Based on the review on traditional vehicle routing problems in various application contexts, we remark that the maintenance time for each WTG/customer takes a significant portion in the overall maintenance time, while traditional vehicle routing problems focus more on minimizing travel time and the service time at each customer is relatively minor as compared to travel time. As such, whether existing optimization models and solution algorithms can be applied to the wind farm disruption recovery operation context remains to be answered. Therefore, it is necessary to investigate how longer maintenance time affects the optimal route pattern, and how existing algorithms for vehicle routing problems can be improved to be fit for the studied problem in this paper.

In summary, the research gap lies in the following two aspects: (1) Current literature on wind farm maintenance operation scheduling mainly aims at minimizing the maintenance travel cost. The loss of wind power generation due to disruption/failure is not considered. However, realworld wind farm operators are concerned about the wind power generation loss and take it as a key criterion for planning the maintenance work. (2) Earlier studies seldom consider the uncertainty in maintenance work, which may cause violation of the working time requirement if longerthan-expected maintenance time of certain WTGs happens. The above two aspects will be verified via examples in detail in Section 5. In view of the research gap, this paper develops an optimization model for the wind farm disruption recovery operation planning problem, which is capable of considering the wind power generation loss and uncertainty in WTG repair time. The proposed optimization model can be used for supporting decision-making of wind farm maintenance work.

\section{Problem Description}

Consider a wind farm with a group of WTGs geographically distributed in an area, and some of the WTGs are detected with a certain failure which requires maintenance/recovery operations to restore normal operating conditions. The wind farm managers need to design a good recovery operation plan in order to facilitate the maintenance team to carry out maintenance activities as efficiently as possible. The disruption recovery operation optimization problem studied in this paper involves two interrelated decisions: (1) routing decision which specifies the sequence of the turbines to be repaired (i.e., maintenance task) for each maintenance team and (2) scheduling decision which details the exact starting time and expected completion time for each maintenance task. Figure 1 shows a real-world example of a wind farm and its maintenance operation plan. As can be seen, there are totally 46 WTGs, nine of them are detected with failure, and two maintenance teams are sent out for conducting the recovery operations. The objective of the maintenance operation planning problem is to minimize the power generation loss of those WTGs with failures. This objective 


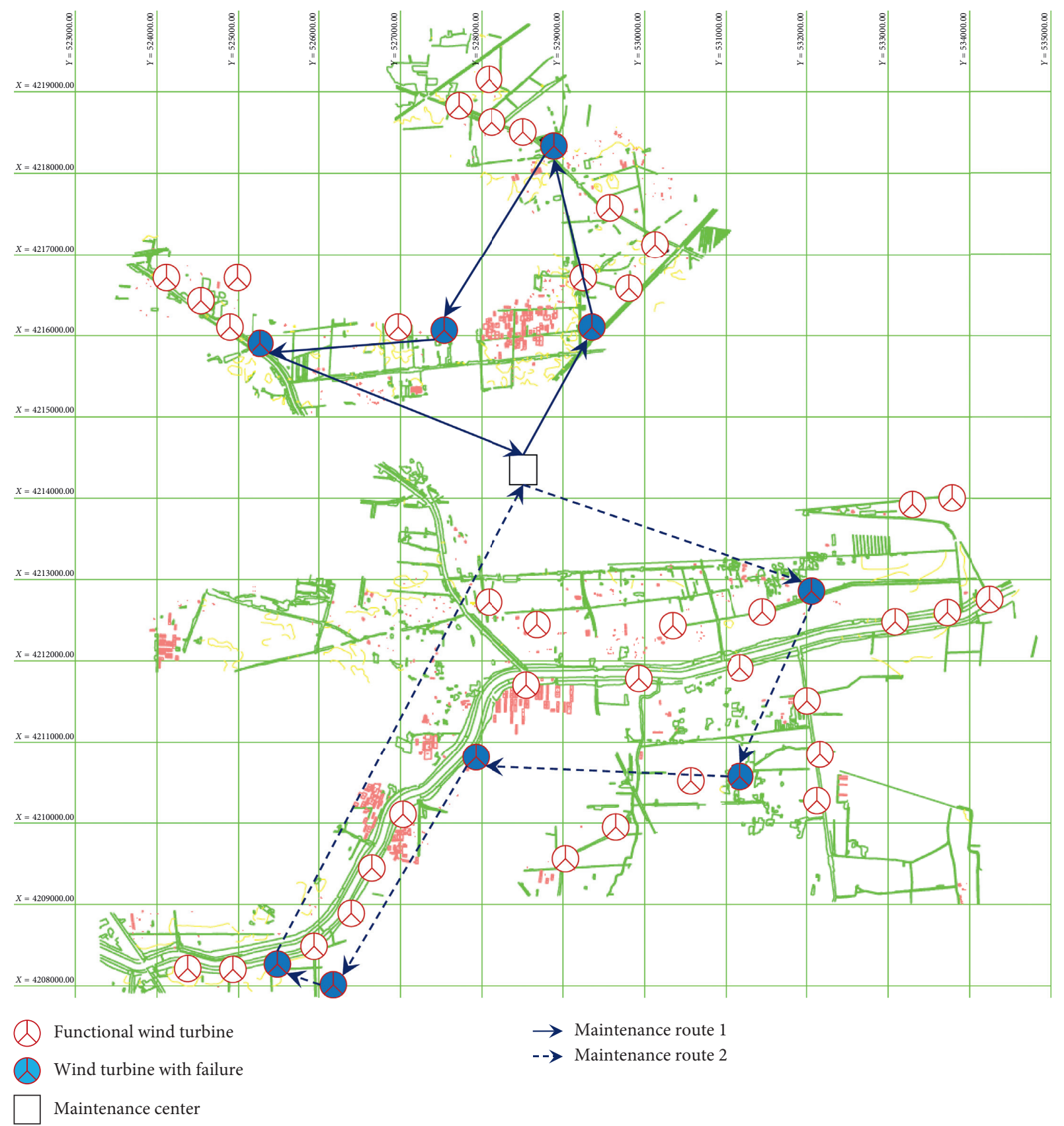

Figure 1: A real-world example of a wind farm and its maintenance operation plan.

could be achieved by determining the optimal sequence and schedule of maintenance operation activities so that the WTGs with failures could be restored to normal operating conditions as fast as possible.

The challenge of the maintenance operation planning problem lies in the following: (1) There are multiple types of failures which could be only restored by maintenance teams with corresponding expertise. This could lead to combinatorial explosion due to multiple failure types and multiple maintenance teams. (2) There are multiple types of WTGs with different power generation capacity, and the power generation capacity of each WTG varies with the change of the real-time wind speed at its location. The uniqueness of the WTGs lies not only in the geographical locations but also in the power generation capacity. This makes the objective of minimizing power generation loss more difficult to achieve. (3) The disruption recovery decision needs to be updated frequently. This requires the developed optimization model to be solved very efficiently. We remark that the studied problem resembles the vehicle routing problem, but it shows unique features. One critical difference lies in the objective function, since the disruption recovery problem for the WTGs aims to minimize the power generation loss rather than the travel cost or distance for traditional vehicle routing problems. This key difference brings a distinct solution structure, which is verified in the following case study. 
The following assumptions are made for the maintenance operation planning problem:

(1) The exact types of failure of those WTGs which need maintenance operations are known. Note that various operating status data are collected automatically by the operating system, and the types of failure could be identified by the real-time data as well as historical data.

(2) The maintenance task of each WTG can be implemented by one maintenance team, and collaboration by multiple teams is not required. In this paper, we do not consider complex maintenance tasks which need more than one team to work on, since such cases are very rare in practice.

\section{Model Formulation and Algorithm}

In this section, we first develop a deterministic mixed integer linear programming model for the studied problem and then propose a chance-constrained programming model in order to take uncertainty of repair time into account. A cuttingplane-based solution algorithm is then designed for obtaining optimal solutions.

4.1. Deterministic Programming Model. The wind farm system can be represented as a graph $G(N, A)$ with WTGs defined as the node set $N$, while road connections between each pair of WTGs defined as the arc set $A$. Based on the defined WTG network, the disruption recovery operation optimization problem is to assign the maintenance tasks to each team and determine the sequence/route and schedule for carrying out the tasks. Before presenting the model, we first introduce the mathematical notations as follows:

Sets

(1) $N$ : set of nodes representing WTGs in the wind farm

(2) $N_{1} \subseteq N$ : set of nodes representing the WTGs with failures

(3) $N_{0}=N \cup\{0\}$ : set of all nodes where 0 represents the maintenance center

(4) A: set of arcs representing the road connection between each pair of WTGs

(5) $K$ : set of maintenance teams

(6) $S$ : set of failure types

Parameters

(1) $\alpha_{\mathrm{ks}} \in\{0,1\}: 1$ if failure type $s$ could be repaired by the maintenance team $k$ or otherwise 0

(2) $\beta_{\text {is }} \in\{0,1\}: 1$ if WTG $i$ needs maintenance for failure type $s$ or otherwise 0

(3) $t_{\mathrm{ij}}$ : relocation time from node $i$ to node $j$

(4) $w_{i}$ : power generation capacity of WTG $i$ under normal conditions

(5) $p_{\text {is }}$ : maintenance time of WTG $i$ for failure type $s$

(6) $T_{\max }$ : maximum working time for each maintenance team
Decision variables

(1) $x_{\mathrm{ijk}} \in\{0,1\}: 1$ if and only if maintenance team $k$ visits WTGs $i$ and $j$ consecutively or otherwise 0

(2) $y_{i} \geq 0$ : recovery time of WTG $i$

Then, the disruption recovery operation planning problem for the WTGs could be formulated as the following mixed integer linear programming (MILP) model (M1):

$$
\min \quad \sum_{i \in N_{1}} w_{i} y_{i}
$$

$$
\begin{aligned}
& \text { subject to: } \quad \sum_{j \in N_{1}} x_{0 \mathrm{jk}}=1, \quad \forall k \in K \text {, } \\
& \sum_{j \in N_{0}} x_{\mathrm{ijk}}-\sum_{j \in N_{0}} x_{\mathrm{jik}}=0, \quad \forall i \in N_{1}, \forall k \in K, \\
& \sum_{i \in N_{1}} x_{i 0 k}=1, \quad \forall k \in K \\
& \sum_{k \in K} \sum_{j \in N_{0}} \sum_{s \in S} \alpha_{\mathrm{ks}} \beta_{\mathrm{is}} x_{\mathrm{ijk}}=1, \quad \forall i \in N, \\
& \sum_{k \in K} \sum_{j \in N_{0}} \sum_{s \in S} \alpha_{\mathrm{ks}} \beta_{\mathrm{is}} x_{\mathrm{jik}}=1, \quad \forall i \in N, \\
& y_{j} \geq t_{0 j}+\sum_{s \in S} \beta_{\mathrm{js}} p_{\mathrm{js}}+M\left(x_{0 \mathrm{jk}}-1\right), \\
& \forall j \in N, \forall i \in K \text {, } \\
& y_{j} \geq y_{i}+t_{\mathrm{ij}}+\sum_{s \in S} \beta_{\mathrm{js}} p_{\mathrm{js}}+M\left(x_{\mathrm{ijk}}-1\right), \\
& \forall i \in N, \forall j \in N, \forall k \in K,
\end{aligned}
$$

$$
\begin{aligned}
& \sum_{j \in N} t_{0 j} x_{0 \mathrm{jk}}+\sum_{i \in N} \sum_{j \in N_{0}}\left(t_{\mathrm{ij}}+\sum_{s} \beta_{\mathrm{is}} p_{\mathrm{is}}\right) x_{\mathrm{ijk}} \leq T_{\max }, \\
& \forall k \in K, \\
& x_{\mathrm{ijk}} \in\{0,1\}, \quad \forall i \in N, \forall j \in N, \forall k \in K, \\
& y_{i} \geq 0, \quad \forall i \in N .
\end{aligned}
$$

The objective function (1) minimizes total recovery time of all WTGs with failures, representing the total power generation loss. Constraints (2)-(4) are the flow conservation constraints. Constraint (2) defines that each maintenance team starts from the maintenance center, while Constraint (4) ensures that each team returns to the center after finishing all the maintenance tasks. For all other nodes, the inflow should equal to the outflow, as is ensured by Constraint (3). Constraints (5) and (6) guarantee for each WTG with failure that exactly one maintenance team visits its location for conducting maintenance works, and its 
corresponding failure matches the expertise of the team. Constraints (7) and (8) define the recovery time for the WTGs with failures. Specifically, Constraint (7) ensures for each maintenance team that the recovery time of its firstvisited WTG is greater than the travel time from the center to its location plus its maintenance time. Similarly, Constraint (8) restricts that, for any two consecutively visited WTGs, the recovery time of the latter one is larger than the summation of that of the former one, the travel time, and the maintenance time of the latter one. Constraint (9) guarantees that, for each maintenance team, the total working time for all the assigned maintenance tasks should respect the maximum working time requirement. Constraints (10) and (11) define the domain of the decision variables.

4.2. Chance-Constrained Programming Model. We further develop a chance-constrained programming model to extend the aforementioned deterministic model to a stochastic setting. Considering that the repair time for WTGs is usually quite uncertain, it is necessary to take the uncertainty into account for the maintenance task assignment and scheduling, especially for ensuring that the working time for each maintenance team does not exceed the total working time limit due to longer-than-expected repair time.

Mathematically, the maintenance time $p_{\text {is }}$ of WTG $i$ for failure type $s$ is treated as a random variable with the mean value of $\mu_{\text {is }}$ and standard deviation of $\sigma_{\text {is }}$. As such, the recovery time $y_{i}$ and the total working time for each maintenance team also become random variables. In the developed constrained programming model, we take the mean value of the recovery time and minimize the expected power generation loss in the objective function. For the working time of maintenance teams, we impose that it does not exceed the maximum working time limit with a least probability $(1-\theta)$. Then, the chance-constrained programming model (M2) could be formulated as follows:

$$
\min \quad \sum_{i \in N_{1}} w_{i} y_{i}
$$

subject to:

$$
\begin{array}{r}
y_{j} \geq t_{0 j}+\sum_{s \in S} \beta_{\mathrm{js}} \mu_{\mathrm{js}}+M\left(x_{0 \mathrm{jk}}-1\right), \\
\forall j \in N, \forall i \in K, \\
y_{j} \geq y_{i}+t_{\mathrm{ij}}+\sum_{s \in S} \beta_{\mathrm{js}} \mu_{\mathrm{js}}+M\left(x_{\mathrm{ijk}}-1\right), \\
\forall i \in N, \forall j \in N, \forall k \in K,
\end{array}
$$

$$
\begin{aligned}
& \operatorname{Pr}\left[\sum_{j \in N} t_{0 j} x_{0 \mathrm{jk}}+\sum_{i \in N} \sum_{j \in N_{0}}\left(t_{\mathrm{ij}}+\sum_{s} \beta_{\mathrm{is}} p_{\mathrm{is}}\right) x_{\mathrm{ijk}} \leq T_{\max }\right] \geq 1-\theta, \\
& \forall k \in K .
\end{aligned}
$$

(2)-(6) and (10), (11)

4.3. A Cutting-Plane-Based Solution Algorithm. Observing that the above chance-constrained programming model involves a nonlinear term in Constraint (15), we develop a cutting-plane-based solution algorithm for solving the model M2 and obtaining optimal solutions. The basic idea of the cutting-plane-based solution algorithm is to relax Constraint (15) by taking the mean value of repair time to impose a loose restriction and solve the updated model which becomes a linear programming model. Once the model is solved, we use the obtained solution and again check Constraint (15). If violation exists, we add new cuts to eliminate such infeasible solutions and rerun the model; otherwise, the optimal solution to model M2 is obtained. The step-by-step algorithm is summarized as follows.

Step 1: replace Constraint (15) by the following:

$$
\begin{aligned}
& \sum_{j \in N} t_{0 j} x_{0 \mathrm{jk}}+\sum_{i \in N} \sum_{j \in N_{0}}\left(t_{\mathrm{ij}}+\sum_{s} \beta_{\mathrm{is}} \mu_{\mathrm{is}}\right) x_{\mathrm{ijk}} \leq T_{\max }, \\
& \forall k \in K .
\end{aligned}
$$

Step 2: solve the updated model (M2) to obtain optimal solutions $\left\{x_{i j k}^{*}\right\}$ and $\left\{y_{i}^{*}\right\}$. Check whether Constraint (15) is violated or not by the following inequality:

$$
\begin{gathered}
\sum_{j \in N} t_{0 j} x_{0 \mathrm{jk}}+\sum_{i \in N} \sum_{j \in N_{0}}\left\{t_{\mathrm{ij}}+\sum_{s} \beta_{\mathrm{is}}\left[\mu_{\mathrm{is}}+\Phi^{-1}(1-\theta) \sigma_{\mathrm{is}}\right]\right\} \\
x_{\mathrm{ijk}} \leq T_{\max }, \quad \forall k \in K,
\end{gathered}
$$

where $\Phi^{-1}(1-\theta)$ is the inverse cumulative probability function with a probability of $(1-\theta)$.

Step 3: if there exists any maintenance team $k$ whose working time limit constraint is violated, add the following cuts:

$$
\sum_{(i, j)||_{i \mathrm{ij}}^{*}=0} x_{\mathrm{ijk}}+\sum_{(i, j) \mid x_{\mathrm{ijk}}^{*}=1}\left(1-x_{\mathrm{ijk}}\right) \geq 1,
$$

and go to Step 2. Otherwise, stop.

\section{Case Study}

We conduct computational experiments based on a realworld wind farm, as is shown in Figure 1, in order to test the effectiveness of the proposed optimization model and solution algorithm. The solution algorithm is coded in $\mathrm{C}++$ and solved by a commercial solver CPLEX.

5.1. Real-World Case and Its Parameters. There are totally 46 WTGs, and ten of them need to be repaired. We consider 10 types of maintenance services and four maintenance teams at the maintenance center. The relationship between the maintenance team's expertise and the failure types is shown in Table 1. As can be seen, the maintenance teams are able to provide service for one or more failure types, but not all. For example, the expertise of maintenance team 1 covers failure types 5 and 10, while 
TABLE 1: Relationship between maintenance teams' expertise and failures.

\begin{tabular}{lcccccccccc}
\hline \multirow{2}{*}{ Maintenance team } & \multicolumn{1}{c}{ Maintenance expertise } & \multicolumn{1}{c}{$c$} & \\
& $s=1$ & $s=2$ & $s=3$ & $s=4$ & $s=5$ & $s=6$ & $s=7$ & $s=8$ & $s=10$ \\
\hline 1 & 0 & 0 & 0 & 0 & 1 & 0 & 0 & 0 & 0 \\
2 & 1 & 0 & 1 & 0 & 0 & 1 & 0 & 0 & 1 \\
3 & 0 & 1 & 1 & 0 & 0 & 0 & 1 & 1 & 0 \\
4 & 0 & 0 & 0 & 1 & 1 & 1 & 0 & 1 & 0 \\
\hline
\end{tabular}

TABLE 2: Types of services and maintenance time required by each faulted WTG.

\begin{tabular}{|c|c|c|c|c|c|c|c|c|c|c|}
\hline \multirow{2}{*}{ WTG } & \multicolumn{10}{|c|}{ Required type of service|required maintenance time $p_{\text {is }}(\min )$} \\
\hline & $s=1$ & $s=2$ & $s=3$ & $s=4$ & $s=5$ & $s=6$ & $s=7$ & $s=8$ & $s=9$ & $s=10$ \\
\hline 1 & $0 \mid 200$ & $1 \mid 250$ & $0 \mid 260$ & $0 \mid 200$ & $1 \mid 200$ & $0 \mid 150$ & $0 \mid 300$ & $0 \mid 210$ & $0 \mid 300$ & $0 \mid 200$ \\
\hline 2 & $0 \mid 200$ & $0 \mid 400$ & $0 \mid 200$ & $0 \mid 200$ & $0 \mid 150$ & $0 \mid 300$ & $1 \mid 210$ & $0 \mid 300$ & $0 \mid 300$ & $0 \mid 200$ \\
\hline 3 & $0 \mid 200$ & $0 \mid 400$ & $1 \mid 260$ & $0 \mid 200$ & $0 \mid 200$ & $0 \mid 150$ & $0 \mid 300$ & $0 \mid 210$ & $0 \mid 300$ & $0 \mid 200$ \\
\hline 4 & $0 \mid 250$ & $0 \mid 450$ & $0 \mid 350$ & $0 \mid 240$ & $0 \mid 260$ & $1 \mid 200$ & $0 \mid 400$ & $1 \mid 260$ & $0 \mid 230$ & $0 \mid 250$ \\
\hline 5 & $1 \mid 200$ & \begin{tabular}{l|l}
0 & 400
\end{tabular} & $0 \mid 260$ & $0 \mid 200$ & $0 \mid 200$ & $0 \mid 150$ & $0 \mid 300$ & $0 \mid 210$ & $0 \mid 300$ & $0 \mid 200$ \\
\hline 6 & $0 \mid 150$ & $0 \mid 300$ & $0 \mid 200$ & $0 \mid 200$ & $0 \mid 150$ & $0 \mid 150$ & $0 \mid 250$ & $0 \mid 200$ & $1 \mid 250$ & $0 \mid 180$ \\
\hline 7 & $0 \mid 200$ & $0 \mid 400$ & $0 \mid 260$ & $1 \mid 250$ & $0 \mid 200$ & $0 \mid 150$ & $0 \mid 300$ & $0 \mid 210$ & $1 \mid 300$ & $0 \mid 200$ \\
\hline 8 & $0 \mid 200$ & $0 \mid 400$ & $0 \mid 260$ & $0 \mid 200$ & $0 \mid 200$ & \begin{tabular}{l|l}
0 & 150
\end{tabular} & $0 \mid 300$ & \begin{tabular}{l|l} 
& 250
\end{tabular} & $1 \mid 300$ & $0 \mid 200$ \\
\hline 9 & $0 \mid 30$ & $0 \mid 250$ & $0 \mid 220$ & $0 \mid 260$ & $0 \mid 300$ & $0 \mid 150$ & $0 \mid 300$ & $0 \mid 210$ & $0 \mid 270$ & $0 \mid 260$ \\
\hline 10 & $0 \mid 200$ & $0 \mid 400$ & $0 \mid 260$ & $0 \mid 200$ & $0 \mid 200$ & $0 \mid 150$ & $0 \mid 300$ & $0 \mid 210$ & $0 \mid 300$ & $1 \mid 200$ \\
\hline
\end{tabular}

TABLE 3: Relocation time between the maintenance center and WTGs.

\begin{tabular}{|c|c|c|c|c|c|c|c|c|c|c|c|}
\hline \multirow{2}{*}{ Maintenance center/WTGs } & \multicolumn{11}{|c|}{ Relocation time $t_{\mathrm{ij}}(\mathrm{min})$} \\
\hline & 0 & 1 & 2 & 3 & 4 & 5 & 6 & 7 & 8 & 9 & 10 \\
\hline 0 & 0 & 100 & 120 & 100 & 110 & 110 & 100 & 200 & 180 & 150 & 100 \\
\hline 1 & 100 & 0 & 90 & 90 & 70 & 70 & 50 & 90 & 110 & 90 & 80 \\
\hline 2 & 12 & 90 & 0 & 80 & 80 & 50 & 60 & 60 & 50 & 70 & 50 \\
\hline 3 & 10 & 90 & 80 & 0 & 50 & 70 & 70 & 50 & 80 & 60 & 50 \\
\hline 4 & 11 & 70 & 80 & 50 & 0 & 60 & 60 & 50 & 50 & 50 & 50 \\
\hline 5 & 11 & 70 & 50 & 70 & 60 & 0 & 70 & 60 & 50 & 40 & 50 \\
\hline 6 & 10 & 50 & 60 & 70 & 60 & 70 & 0 & 60 & 60 & 50 & 50 \\
\hline 7 & 20 & 90 & 60 & 50 & 50 & 60 & 60 & 0 & 40 & 50 & 80 \\
\hline 8 & 18 & 11 & 50 & 80 & 50 & 50 & 60 & 40 & 0 & 60 & 90 \\
\hline 9 & 15 & 90 & 70 & 60 & 50 & 40 & 50 & 50 & 60 & 0 & 70 \\
\hline 10 & 100 & 80 & 50 & 50 & 50 & 50 & 50 & 80 & 90 & 70 & 0 \\
\hline
\end{tabular}

TAble 4: Power generation capacity of WTGs under normal conditions (kW).

\begin{tabular}{|c|c|c|c|c|c|c|c|c|c|c|}
\hline \multirow{2}{*}{ Power generation capacity } & \multicolumn{10}{|c|}{ WTGs } \\
\hline & 1 & 2 & 3 & 4 & 5 & 6 & 7 & 8 & 9 & 10 \\
\hline$w_{i}$ & 2200 & 2200 & 2200 & 2300 & 2200 & 2000 & 2200 & 2200 & 2200 & 2200 \\
\hline
\end{tabular}

maintenance team 4 is able to provide five types of services. This increases the decision difficulty for arranging the maintenance tasks. Table 2 reports the types of failures and the required maintenance time for the ten WTGs. The relocation time between the maintenance center and the WTG or any two WTGs is provided in Tables 3 and 4 shows the power generation capacity of WTGs under normal conditions.

5.2. Optimal Maintenance Plan vs. Existing Approach. By solving the proposed MILP model with CPLEX, the optimal disruption recovery operation plan with least power generation loss could be obtained. The optimal solution could be obtained within 30 minutes. Figure 2 shows the optimal maintenance task assignment and route for the four maintenance teams. As can be seen, maintenance teams 1 and 4 cover the four WTGs needing maintenance tasks in the upper part area, and each of them is responsible for repairing two WTGs. The remaining two teams provide services for the remaining six WTGs in the lower part area, and each of them covers three WTGs. In order to validate the proposed approach, we further treat this problem as a traditional vehicle routing problem and optimize the maintenance routes with the objective of minimizing total 


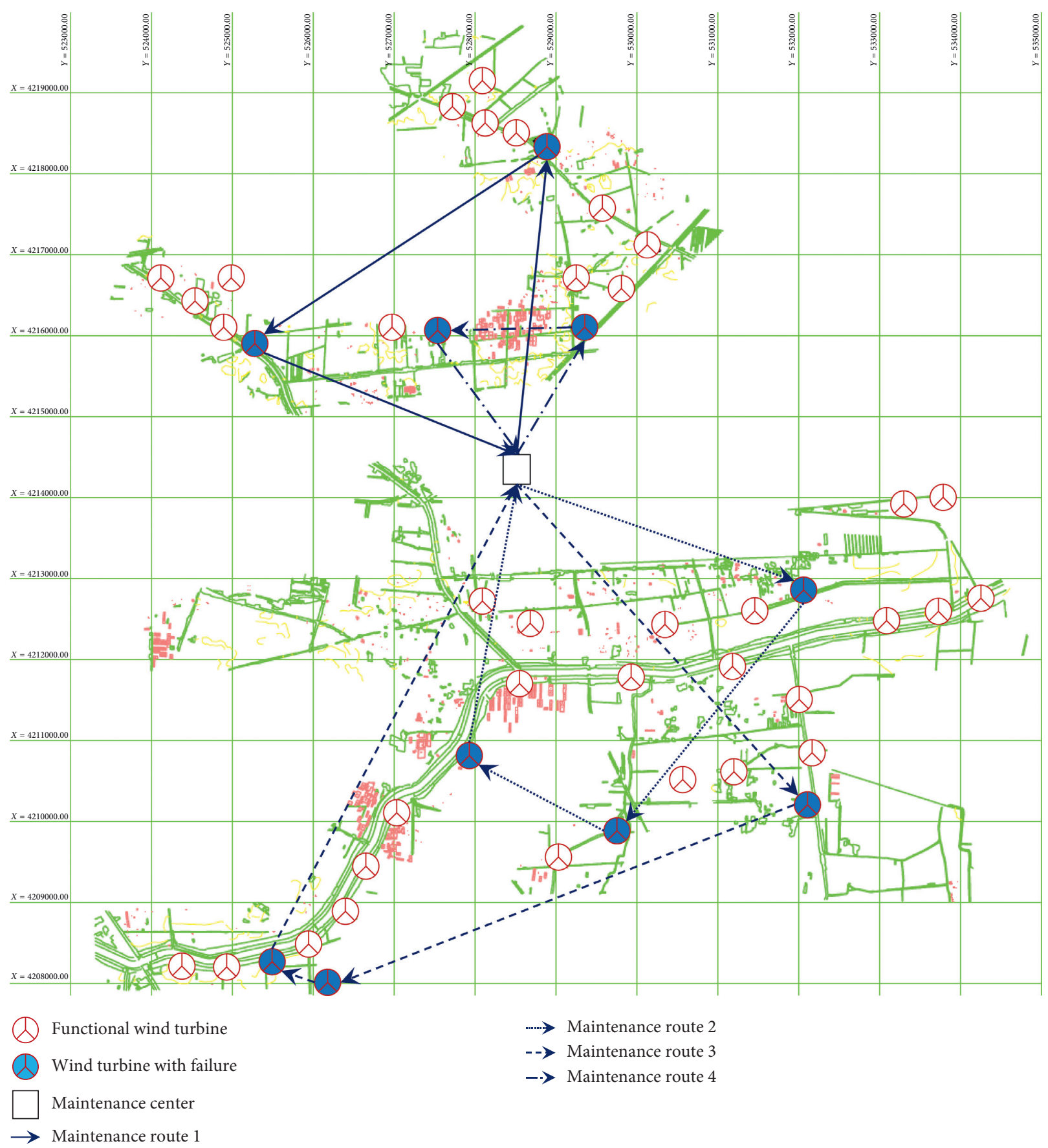

FIGURE 2: Optimal maintenance task assignment and routes obtained from the proposed model.

travel time. The classical sweep algorithm is employed, and the maintenance routes are presented in Figure 3.

By comparing the optimal routing pattern of the proposed recovery operation planning problem and that of the traditional vehicle routing problem, we note that the routes of the maintenance teams of the proposed problem show crossing patterns with each other, while the routes do not cross each other for the vehicle routing problem. For example, the route of maintenance team 2 crosses that of maintenance team 3. This feature is very different from the vehicle routing problem which seldom exhibits crossing patterns. We remark that this is attributed to the structure of the objective function. Specifically, the vehicle routing problems aim to minimize the total travel cost (measured by time or distance), while the recovery operation planning problem for wind farms is to minimize the recovery time of all WTGs. This difference brings out the crossing pattern for the maintenance routes and makes it inapplicable to use traditional algorithms for vehicle routing problems, such as the sweep algorithm. In addition, we also compare the total objective function value and the working time obtained by the two 


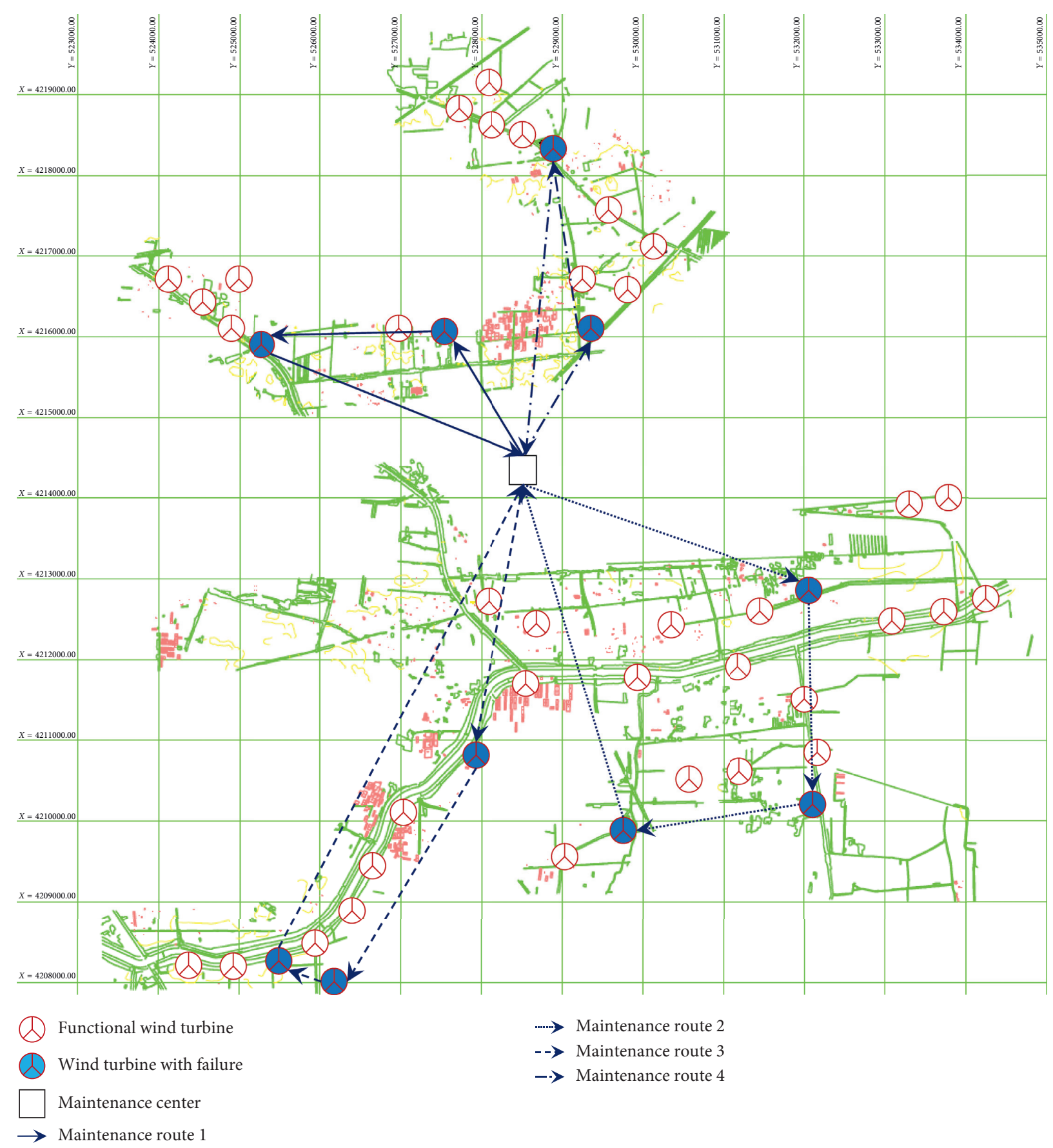

FIGURE 3: Optimal maintenance task assignment and routes based on the sweep algorithm for the vehicle routing problem.

TABLE 5: Comparison of the optimal maintenance plan of the proposed recovery planning problem and that of the traditional vehicle routing problem.

\begin{tabular}{lcccc}
\hline Approach & Maintenance teams & Objective function value & Working time requirement & Improvement \\
\hline Vehicle routing problem & 4 & $1.58 \times 10^{\wedge}$ & Not satisfied & NA \\
Proposed recovery planning problem & 4 & $1.12 \times 10^{\wedge} 7$ & Satisfied & $29.1 \%$ \\
\hline
\end{tabular}

approaches, as is presented in Table 5 . As can be seen, although both approaches require four maintenance teams, the overall recovery time of the recovery planning problem is $29.1 \%$ less, and the traditional vehicle routing problem fails to satisfy the working time requirement. Therefore, we can conclude that the maintenance time for WTGs should be considered in the objective function, and the proposed MILP model is capable of generating 
TABLE 6: Computational results of synthetic experiments.

\begin{tabular}{|c|c|c|c|c|}
\hline Instance & WTGs & Maintenance teams & Objective function value & Time (sec) \\
\hline 1 & 2 & 2 & $6.1 \times 10^{\wedge} 5$ & 0.1 \\
\hline 2 & 4 & 2 & $2.8 \times 10^{\wedge} 6$ & 129 \\
\hline 3 & 6 & 2 & $4.9 \times 10^{\wedge} 6$ & 850 \\
\hline 4 & 8 & 3 & $7.2 \times 10^{\wedge} 6$ & 1604 \\
\hline 5 & 10 & 3 & $9.6 \times 10^{\wedge} 6$ & 1863 \\
\hline 6 & 12 & 3 & $1.3 \times 10^{\wedge} 7$ & 2450 \\
\hline 7 & 14 & 4 & $1.5 \times 10^{\wedge} 7$ & 2685 \\
\hline 8 & 16 & 4 & $1.8 \times 10^{\wedge} 7$ & 3245 \\
\hline
\end{tabular}

TABLE 7: Computational results under different uncertainty levels.

\begin{tabular}{lcccc}
\hline Instance & WTGs & Maintenance teams & Uncertainty level (\%) & No. of cuts generated \\
\hline 1 & 10 & 3 & 5 & 2 \\
2 & 10 & 3 & 10 & 5 \\
3 & 10 & 3 & 15 & 7 \\
4 & 10 & 3 & 20 & 11 \\
5 & 10 & 4 & 25 & 14 \\
6 & 10 & 4 & 30 & 15 \\
\hline
\end{tabular}

optimal maintenance task assignment as well as routing and scheduling decisions for the maintenance teams.

5.3. Synthetic Computational Experiments. We further conduct computational experiments based on the real-world wind farm setting but with different numbers of WTGs needing repair (ranging from 2 to 16) and different numbers of maintenance teams (ranging from 2 to 4). Table 6 reports the computational results including the optimal objective function value and computational time. As can be seen, all the test instances could be solved by the proposed cutting-plane-based solution algorithm within one-hour computational time, and the proposed algorithm shows satisfactory performance.

5.4. Effectiveness of considering Uncertainty of Repair Time. In order to check the necessity of considering the uncertainty of repair time, we further run computational experiments under different uncertainty levels. The uncertainty level is defined as the ratio of standard deviation and mean value. In this study, we consider the uncertainty level ranging from $5 \%$ to $30 \%$. Table 7 reports the computational results showing the relationship between the uncertainty level and the number of cuts generated. As can be seen, even under a small uncertainty level (5\%), two cuts are generated. With higher uncertainty levels, the number of cuts generated increases. This shows that if the uncertainty of repair time is not considered, it is quite possible that the total working time of maintenance teams could exceed the working time limit requirement. Therefore, it is necessary to take uncertainty of repair time into account when planning the disruption recovery operations, and our proposed model and solution algorithm could effectively solve the studied problem.

\section{Conclusions and Future Work}

In this paper, we investigated the disruption recovery operation planning problem arising from wind farms. We firstly developed a mixed integer linear programming model for the problem with the objective of minimizing total power generation loss and then proposed a chanceconstrained programming model as well as a cuttingplane-based solution algorithm to account for the repair time uncertainty. A case study based on a real-world wind turbine power generation farm showed that the proposed model and algorithm are capable of obtaining the optimal maintenance task assignment, as well as the exact route and schedule for each maintenance team. By comparing it with the traditional vehicle routing problem with similar characteristics, the findings demonstrated that the maintenance routes of the studied recovery operation planning problem exhibit unique crossing patterns. By modeling the WTG repair time as a random variable and adding constraints iteratively if longer-than-expected repair time happens, we demonstrated that the proposed cutting-plane algorithm is effective in generating maintenance plans while satisfying the working time limit requirement. This research proposes a novel methodology for addressing the disruption recovery operation planning problem with uncertain repair time. The proposed model and algorithm provide decision-making support for wind farm maintenance works and show a great potential in wind farm energy management.

Finally, a promising direction for future research is to use weather prediction information and consider power generation efficiency under different wind conditions. The existing models can be extended to integrate this consideration to further reduce power generation loss. 


\section{Data Availability}

The data used to support the findings of this study are available from the corresponding author upon request.

\section{Conflicts of Interest}

The authors declare that there are no conflicts of interest regarding the publication of this paper.

\section{Acknowledgments}

This research was funded by the Science and Technology Foundation of Hebei Province (16214319), Shanghai RisingStar Program and Shanghai Municipal Science and Technology Commission (17DZ1203300).

\section{References}

[1] Z. Chen and F. Blaabjerg, "Wind farm-a power source in future power systems," Renewable and Sustainable Energy Reviews, vol. 13, no. 6-7, pp. 1288-1300, 2009.

[2] Z. Chen, J. M. Guerrero, and F. Blaabjerg, "A review of the state of the art of power electronics for wind turbines," IEEE Transactions on Power Electronics, vol. 24, no. 8, pp. 18591875, 2009.

[3] B. K. Sahu, "Wind energy developments and policies in China: a short review," Renewable and Sustainable Energy Reviews, vol. 81, pp. 1393-1405, 2018.

[4] Z. W. Geem and J. Hong, "Improved formulation for the optimization of wind turbine placement in a wind farm," Mathematical Problems in Engineering, vol. 2013, Article ID 481364, 5 pages, 2013.

[5] M. Fischetti and D. Pisinger, "Optimizing wind farm cable routing considering power losses," European Journal of Operational Research, vol. 270, no. 3, pp. 917-930, 2018.

[6] N. Haouas and P. R. Bertrand, "Wind farm power forecasting," Mathematical Problems in Engineering, vol. 2013, Article ID 163565, 5 pages, 2013.

[7] K. Pan, Z. Qian, and N. Chen, "Probabilistic short-term wind power forecasting using sparse Bayesian learning and NWP," Mathematical Problems in Engineering, vol. 2015, Article ID 785215, 11 pages, 2015.

[8] Z. Zhang, J. Li, and P. Zhuge, "Failure analysis of large-scale wind power structure under simulated typhoon," Mathematical Problems in Engineering, vol. 2014, Article ID 486524, 10 pages, 2014.

[9] F. Deng and Z. Zhe Chen, "Operation and control of a DCgrid offshore wind farm under DC transmission system faults," IEEE Transactions on Power Delivery, vol. 28, no. 3, pp. 1356-1363, 2013.

[10] M. Shafiee, "Maintenance logistics organization for offshore wind energy: current progress and future perspectives," Renewable Energy, vol. 77, pp. 182-193, 2015.

[11] J.-H. Huh and K. Seo, "Hybrid advanced metering infrastructure design for micro grid using the game theory model," International Journal of Software Engineering and Its Applications, vol. 9, no. 9, pp. 257-268, 2015.

[12] J. C. Kim, J. H. Huh, and J. S. Ko, "Improvement of MPPT control performance using fuzzy control and VGPI in the PV system for micro grid," Sustainability, vol. 11, no. 21, p. 5891, 2019.
[13] S. Perez-Canto and J. C. Rubio-Romero, "A model for the preventive maintenance scheduling of power plants including wind farms," Reliability Engineering \& System Safety, vol. 119, pp. 67-75, 2013.

[14] M. Stålhane, L. M. Hvattum, and V. Skaar, "Optimization of routing and scheduling of vessels to perform maintenance at offshore wind farms," Energy Procedia, vol. 80, pp. 92-99, 2015.

[15] L. Dai, M. Stålhane, and I. B. Utne, "Routing and scheduling of maintenance fleet for offshore wind farms," Wind Engineering, vol. 39, no. 1, pp. 15-30, 2015.

[16] C. A. Irawan, D. Ouelhadj, D. Jones, M. Stålhane, and I. B. Sperstad, "Optimisation of maintenance routing and scheduling for offshore wind farms," European Journal of Operational Research, vol. 256, no. 1, pp. 76-89, 2017.

[17] B. Bao and T. Li, "Equipment maintenance operations scheduling method with random machine breakdowns," Computer Engineering and Applications, vol. 53, no. 9, pp. 258-262, 2017.

[18] S. Lan, J.-P. Clarke, and C. Barnhart, "Planning for robust airline operations: optimizing aircraft routings and flight departure times to minimize passenger disruptions," Transportation Science, vol. 40, no. 1, pp. 15-28, 2006.

[19] J. G. Jin, K. M. Teo, and A. R. Odoni, "Optimizing bus bridging services in response to disruptions of urban transit rail networks," Transportation Science, vol. 50, no. 3, pp. 790-804, 2016.

[20] F. Liberatore, M. T. Ortuño, G. Tirado, B. Vitoriano, and M. P. Scaparra, "A hierarchical compromise model for the joint optimization of recovery operations and distribution of emergency goods in Humanitarian Logistics," Computers \& Operations Research, vol. 42, pp. 3-13, 2014.

[21] P. Grangier, M. Gendreau, F. Lehuédé, and L.-M. Rousseau, "An adaptive large neighborhood search for the two-echelon multiple-trip vehicle routing problem with satellite synchronization," European Journal of Operational Research, vol. 254, no. 1, pp. 80-91, 2016.

[22] G. Ćirović, D. Pamučar, and D. Božanić, "Green logistic vehicle routing problem: routing light delivery vehicles in urban areas using a neuro-fuzzy model," Expert Systems with Applications, vol. 41, no. 9, pp. 4245-4258, 2014.

[23] L. Zhou, R. Baldacci, D. Vigo, and X. Wang, "A multi-depot two-echelon vehicle routing problem with delivery options arising in the last mile distribution," European Journal of Operational Research, vol. 265, no. 2, pp. 765-778, 2018.

[24] M. Christiansen, E. Hellsten, D. Pisinger, D. Sacramento, and C. Vilhelmsen, "Liner shipping network design," European Journal of Operational Research, 2019, In press.

[25] K. H. Kjeldsen, "Classification of ship routing and scheduling problems in liner shipping," INFOR: Information Systems and Operational Research, vol. 49, no. 2, pp. 139-152, 2011.

[26] E. Wibisono and P. Jittamai, "Multi-objective evolutionary algorithm for a ship routing problem in maritime logistics collaboration," International Journal of Logistics Systems and Management, vol. 28, no. 2, pp. 225-252, 2017.

[27] C. Fikar and P. Hirsch, "Home health care routing and scheduling: a review," Computers \& Operations Research, vol. 77, pp. 86-95, 2017.

[28] D. S. Mankowska, F. Meisel, and C. Bierwirth, "The home health care routing and scheduling problem with interdependent services," Health Care Management Science, vol. 17, no. 1, pp. 15-30, 2014.

[29] S. V. Begur, D. M. Miller, and J. R. Weaver, "An integrated spatial DSS for scheduling and routing home-health-care nurses," Interfaces, vol. 27, no. 4, pp. 35-48, 1997. 